\title{
Avaliação de diferentes metodologias para obtenção do Módulo de Resiliência de misturas asfálticas
}

\author{
Raul Serafim Ponte1, Verônica Teixeira Franco Castelo Branco², \\ Áurea Silva de Holanda ${ }^{3}$, Jorge Barbosa Soares ${ }^{4}$
}

\begin{abstract}
Resumo: O Módulo de Resiliência (MR) é um dos principais parâmetros utilizados para o dimensionamento empírico-mecanístico de pavimentos asfálticos no Brasil. Foi publicada recentemente no Brasil uma nova norma para a obtenção desse parâmetro, a NBR 16018 (2011) da ABNT, baseada nas principais normativas internacionais para o ensaio. Devido à existência de divergências nas metodologias de ensaio entre as normativas brasileiras atuais, foram realizados ensaios para obtenção do MR em laboratório utilizando diferentes metodologias. Foram avaliadas três misturas distintas do tipo Concreto Asfáltico (CA). Observou-se pouca variação na média dos resultados de MR obtidos em laboratório. O impacto da diferença obtida nos resultados dos ensaios realizados foi avaliado por meio da análise de tensões e de deformações de uma estrutura de pavimento asfáltico com o auxílio do software CAP3D. A partir desta análise, pode-se observar que, para a estrutura avaliada, também houve pouca influência da metodologia utilizada para a obtenção do MR nos resultados obtidos.
\end{abstract}

Palavras-chave: Misturas Asfálticas. Módulo de Resiliência. Metodologia.

\begin{abstract}
The Resilient Modulus (RM) is one of the main parameters used on the mechanistic-empirical asphalt pavement design in Brazil. It was recently published in Brazil a new standard for determining this parameter, the NBR 16018 (2011) from $A B N T$, based on the main international standards. Due to the existence of differences in testing methodology between the current Brazilian standards, tests were carried out to obtain MR in the laboratory using different methodologies. Three different mixtures of Asphalt Concrete (AC) were evaluated. It was observed that there was little variation in the average of the MR results. The impact of the difference in the results obtained from laboratory tests was assessed by means of a structural analysis of an asphalt pavement using the computational program CAP3D. From the analysis, it was observed for the structure examined, that there was also little influence of the methodology used to obtain the MR on the structural response of the layered system.
\end{abstract}

Keywords: Hot Mix Asphalt. Resilient Modulus. Testing Methodology.

\section{INTRODUÇÃO}

O parâmetro rigidez tornou-se essencial para o dimensionamento de pavimentos, após o desenvolvimento dos métodos mecanísticos, que compatibilizam a solicitação do tráfego com dados de resistência dos materiais (Franco, 2007). Pode-se citar os métodos de Kerkhoven e Dormon (1953), Saal e Pell (1960) e Dormon e Metcalf (1965), métodos nacionais como o Método da Resiliência (Motta, 1991), além de métodos de dimensionamento que utilizam o

\footnotetext{
1 Universidade Federal do Ceará, Departamento de Engenharia de Transportes, Laboratório de Mecânica dos Pavimentos (rpserafim@gmail.com).

2 Universidade Federal do Ceará, Departamento de Engenharia de Transportes, Laboratório de Mecânica dos Pavimentos (veronica@det.ufc.br).

3 Universidade Federal do Ceará, Departamento de Engenharia de Transportes, Laboratório de Mecânica dos Pavimentos (aurea@det.ufc.br).

4 Universidade Federal do Ceará, Departamento de Engenharia de Transportes, Laboratório de Mecânica dos Pavimentos (jsoares@det.ufc.br).
}

Manuscrito recebido em 22/05/2014 e aprovado para publicação em 01/07/2014. Este artigo é parte de TRANSPORTES v. 22, n. 2, 2014. ISSN: 2237-1346 (online).

DOI: http://dx.doi.org/10.14295/transportes.v22i2.792.
Módulo Dinâmico (MD) como parâmetro de rigidez, como por exemplo o Mechanistic-Empirical Pavement Design Guide da American Association of State Highway and Transportation Officials (AASHTO) (Bernucci et al., 2007; Araújo et al., 2009; Soares et al., 2009).

O MR foi introduzido por Francis Hveem em 1955, como forma de melhor representar o módulo de elasticidade para materiais sob carregamentos cíclicos. De acordo com a teoria da elasticidade, tanto o MR quanto o módulo de elasticidade possuem o mesmo conceito, sendo ambos representados pela razão entre a tensão e a deformação. A motivação para a diferenciação entre estes, foi as discrepâncias entre a ordem de grandeza de deslocamentos dos materiais utilizados em pavimentação em relação aos materiais elásticos comumente utilizados na engenharia (Brito, 2006).

A análise dos resultados de MR não permite uma modelagem apropriada dos fenômenos comumente associados aos pavimentos, por não considerar o comportamento viscoelástico do mesmo. Porém, este ainda é o parâmetro de rigi- 
dez mais difundido no país, devido tanto à cultura de utilização desse parâmetro quanto ao alto valor de aquisição de equipamentos para obtenção do MD. Para a realidade brasileira, o ensaio de MR é, numa análise comparativa, avançado quando considera-se a caracterização mecânica dos materiais exigida pelo método de dimensionamento em vigor no país, que ainda baseia-se em resultados do ensaio de CBR (California Bearing Ratio ou Índice de Suporte Califórnia), bem como no uso do número $N$.

Variações nos parâmetros de ensaio podem levar à inconsistência de resultados caso utilizem-se metodologias distintas, pois os resultados de MR mostram alta sensibilidade às metodologias de cálculo, à frequência de ensaio, à temperatura e ao pulso de carga, sendo a magnitude desta influência função das propriedades das misturas asfálticas avaliadas (Brito, 2006). Verifica-se que algumas normas não são suficientemente específicas em relação a alguns desses parâmetros citados, podendo levar a problemas relacionados à repetibilidade, mesmo em casos onde o mesmo procedimento é utilizado. Essas divergências nos parâmetros podem levar a resultados que acarretem diferenças na análise (tensões e deformações) e no dimensionamento de estruturas. Tais diferenças podem afetar aspectos como: custo, vida útil e segurança dos pavimentos.

O presente trabalho tem como objetivo avaliar o impacto da utilização de diferentes metodologias de ensaio para obtenção em laboratório de MR para misturas asfálticas, a influência do uso de diferentes materiais e de curvas granulométricas distintas. Além de que, espera-se verificar o impacto dos resultados obtidos na análise de tensões e de deformações de uma estrutura de pavimento asfáltico.

\section{MATERIAIS E MÉTODOS}

\subsection{Materiais utilizados}

Neste trabalho foram utilizados dois CAPs: convencional e modificado. O CAP convencional foi fornecido pela Lubnor/Petrobras e é classificado por penetração como 50/70 e de acordo com a classificação Superpave como PG
70-28 (Coutinho, 2012). O mesmo ligante foi modificado com a adição de $4 \%$ em massa de EVA, a partir do estudo desenvolvido por Alencar (2009). A modificação do CAP foi realizada com o auxílio de um misturador de baixo cisalhamento e hélice cisalhante da marca Fisatom®, nas seguintes condições: temperatura de $160 \pm 5^{\circ} \mathrm{C}$ e rotação de $544 \mathrm{rpm}$ por um período de 2 horas.

Os agregados minerais utilizados têm suas jazidas localizadas na Região Metropolitana de Fortaleza (RMF), especificamente nos munícipios de Itaitinga, Eusébio, Caucaia e Maracanaú, e são comumente empregados em obras de pavimentação no estado. Os materiais são de origem granítica, gnáissica e fonolítica (Bessa, 2012). A areia de campo utilizada, proveniente do sítio Maninho, foi cedida pela Insttale Engenharia Ltda. A caracterização dos materiais e as dosagens foram realizadas no estudo de Coutinho (2012).

Neste trabalho foram avaliadas três misturas asfálticas, com duas curvas granulométricas distintas, ambas incluídas na faixa $\mathrm{C}$ para $\mathrm{CAs}$ do DNIT (DNIT ES 031, 2006). Para a Mistura 1, utilizou-se como agregado graúdo as britas $1 / 2$ " e 3/8" (ambas de origem fonolítica), pó de pedra (origem gnáissica) e areia de campo, nas proporções de $20 \%, 30 \%$, $25 \%$ e $25 \%$, respectivamente. Para as Misturas 2 e 3, foram utilizadas as britas 3/4" e 3/8", como agregado graúdo, e como agregado miúdo, pó de pedra, com proporções de $20 \%$, 44\% e $36 \%$, respectivamente. Todos os materiais são de origem granítica. As curvas granulométricas utilizadas estão representadas na Figura 1.

$\mathrm{O}$ teor de projeto de CAP definido por Coutinho (2012), através da metodologia Superpave, para que se atingisse um volume de vazios (Vv) de 4\% foi: (i) Mistura 1-5,0\%, (ii) Mistura $2-5,5 \%$ e (iii) Mistura $3-5,7 \%$. Foram replicados cinco CPs para cada mistura para a realização dos ensaios mecânicos, sendo realizado o procedimento de envelhecimento de curto prazo em estufa antes da compactação de todas as amostras. Admitiu-se uma variação de $0,4 \%$ no $\mathrm{Vv}$ para mais ou para menos para as amostras ensaiadas. 


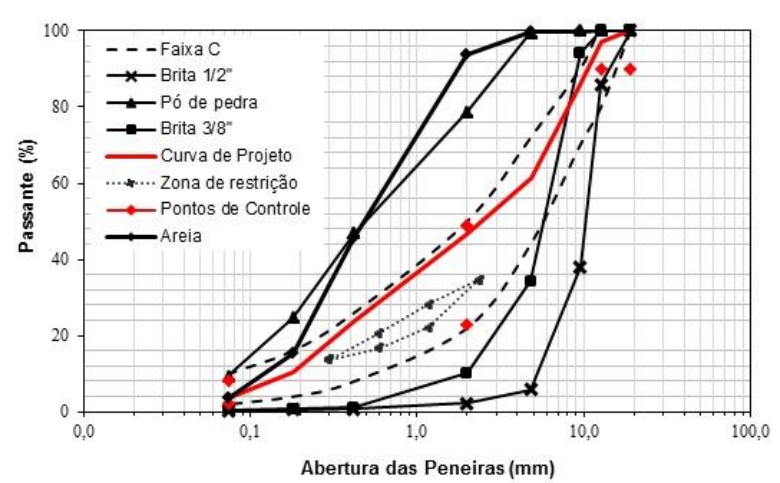

(a)

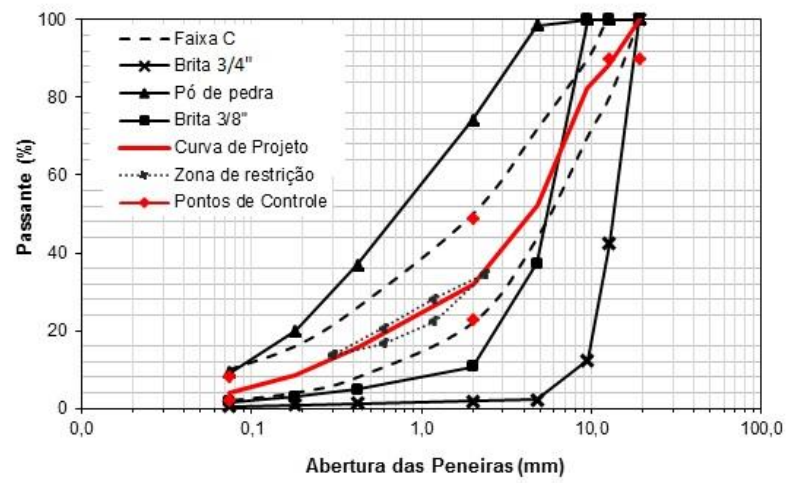

(b)

Figura 1 - Curvas granulométricas utilizadas - Mistura 1 (a), Misturas 2 e 3 (b)

\subsection{Escolha das metodologias de ensaio de MR}

Para a realização desse trabalho, fez-se uma investigação das principais normas nacionais e internacionais para a obtenção do MR para misturas asfálticas em laboratório. Optouse por verificar as normas mais referenciadas na literatura, as citadas como referência nos manuais dos equipamentos utilizados para o ensaio, como também, a nova norma brasileira para obtenção do MR de misturas asfálticas, a ABNT NBR 16018 (2011). As normas investigadas foram: (i) BSi DD 213(1993) - Inglaterra, (ii) DNER ME 133(1994) - Brasil, (iii) ASTM D 7369 (2009) - Estados Unidos, (iv) AS 2891.13.1 (1995) - Austrália, (v) AASHTO TP31(1996) - Estados Unidos, (vi) EN 1296726 (2004) - Europa, (vii) DNIT ME 135 (2010) - Brasil e (viii) ABNT NBR 16018 (2011) Brasil.

No estudo dessas normas, fez-se um levantamento das recomendações descritas pelas mesmas sobre os parâmetros que, acredita-se, desempenham maior influência nos resultados obtidos no ensaio de MR. Quais sejam: tipo de carregamento (hidráulico ou pneumático), frequência de ensaio, número de ciclos na fase de pré-condicionamento, número de ciclos na fase de condicionamento, número de ciclos utilizados para o cálculo do MR, temperatura de ensaio, período mínimo de estabilização da temperatura, utilização de faixas de temperaturas, coeficiente de Poisson (determinado ou assumido), método para o cálculo do MR total, método para o cálculo do MR instantâneo.

Após a análise das principais metodologias, optou-se por utilizar as metodologias brasileiras DNER ME 133 (1994) e a ABNT NBR
16018 (2011). Além destas, comparou-se os resultados com os obtidos diretamente do software do equipamento pneumático de fabricação da empresa SIGEO. A razão dessa escolha é a existência de um extenso banco de dados de resultados de MR obtidos no Laboratório de Mecânica dos Pavimentos (LMP) da Universidade Federal do Ceará (UFC) com o uso deste equipamento.

A escolha da metodologia DNER ME 133 (1994), mais antiga, em detrimento da DNIT ME 135 (2010), se deu devido à baixa especificidade da norma mais atual em relação a diversos parâmetros de ensaio, por exemplo: número de ciclos utilizados na fase de pré-condicionamento, número de ciclos utilizados na fase de condicionamento, escolha dos ciclos utilizados para o cálculo do MR, período de estabilização da temperatura de ensaio, entre outros. A baixa especificidade desses diversos parâmetros deixam muitas decisões a cargo do operador, podendo impactar os resultados obtidos. A norma ABNT NBR 16018 (2011) foi escolhida para verificar o impacto da nova metodologia nos resultados de MR obtidos no Brasil. Esta norma se encontra em alinhamento com as recomendações das principais normas internacionais.

As recomendações de ensaio do fabricante do equipamento pneumático SIGEO encontramse no manual do usuário do produto (SICAEP, 2004). Algumas adaptações foram feitas, como por exemplo, no número de ciclos de condicionamento, baseadas em contatos pessoais com os técnicos da empresa em visita ao LMP. As principais recomendações de cada uma das metodologias escolhidas para a realização dos ensaios neste trabalho estão resumidas na Tabela 1. 
Tabela 1 - Principais recomendações das metodologias selecionadas

\begin{tabular}{|c|c|c|c|}
\hline $\begin{array}{l}\text { Procedimento/ } \\
\text { Parâmetro }\end{array}$ & $\begin{array}{c}\text { ABNT NBR } 16018 \\
(2011)\end{array}$ & $\begin{array}{l}\text { DNER ME } 133 \\
(1994)\end{array}$ & $\begin{array}{c}\text { SIGEO } \\
\text { (SICAEP, 2004) }\end{array}$ \\
\hline $\begin{array}{c}\text { Tipo de carrega- } \\
\text { mento }\end{array}$ & Hidráulico/ Pneumático & Pneumático & Pneumático \\
\hline Frequência (Hz) & 1 & 1 & 1 \\
\hline $\begin{array}{l}\text { Ciclos de pré- } \\
\text { condicionamento }\end{array}$ & 50 & $\begin{array}{l}\text { - } 200 \text { ciclos ou até a obtenção } \\
\text { de registro mensurável no os- } \\
\text { cilógrafo } \\
\text { - Carregamento máximo } \rightarrow \\
30 \% \text { da RT }\end{array}$ & $\begin{array}{l}\text { - Incrementos de carga } \\
\text { até a obtenção de um re- } \\
\text { gistro mensurável no osci- } \\
\text { lógrafo }\end{array}$ \\
\hline $\begin{array}{l}\text { Ciclos de condiciona- } \\
\text { mento }\end{array}$ & 15 & 500 & 60 \\
\hline $\begin{array}{c}\text { Ciclos para o cálculo } \\
\text { do MR }\end{array}$ & $\begin{array}{c}15 \text { (divididos em } 3 \text { grupos } \\
\text { de } 5 \text { ciclos cada) }\end{array}$ & $\begin{array}{c}3 \text { (para os ciclos de no: } 300, \\
400 \text { e } 500)\end{array}$ & $\begin{array}{c}15 \text { (divididos em } 3 \text { grupos } \\
\text { de } 5 \text { ciclos cada) }\end{array}$ \\
\hline Temperatura $\left({ }^{\circ} \mathrm{C}\right)$ & 25 & 30 & 25 \\
\hline $\begin{array}{l}\text { Período de estabiliza- } \\
\text { ção da temperatura }\end{array}$ & 4 h (mínimo) & Não especifica & Não especifica \\
\hline Coef. de Poisson & $\begin{array}{c}\text { Determinado / Assumido } \\
(\mathrm{v}=0,30)\end{array}$ & $\begin{array}{l}\text { Assumido } \\
(v=0,30)\end{array}$ & $\begin{array}{l}\text { Assumido } \\
(\mathrm{v}=0,30)\end{array}$ \\
\hline Cálculo do MR Total & Sim & Não & Não \\
\hline $\begin{array}{l}\text { Cálculo do MR Ins- } \\
\text { tantâneo }\end{array}$ & Sim & Sim & Sim \\
\hline
\end{tabular}

\subsection{Ensaios de MR}

Os ensaios de MR foram realizados seguindo as metodologias previamente selecionadas utilizando uma prensa pneumática. Os deslocamentos foram medidos por dois LVDTs (Linear Variable Displacement Transducers) posicionados na lateral dos CPs de forma a captar as deformações no plano perpendicular à aplicação da carga. Para o posicionamento cor-

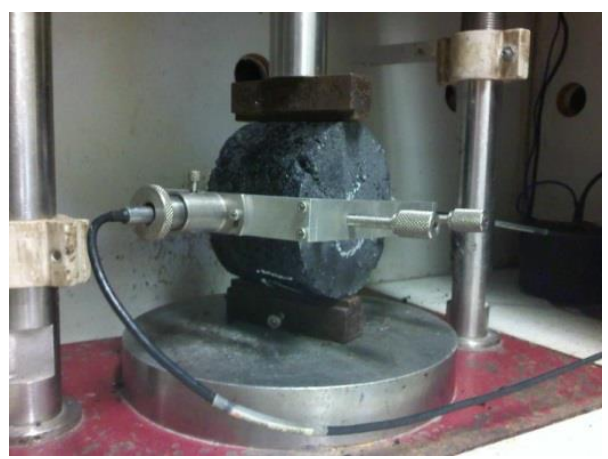

(a) reto da estrutura que suporta os LVDTs, de forma a captar as deformações no diâmetro máximo do $\mathrm{CP}$, utilizou-se um gabarito para a montagem do ensaio, para CPs de $100 \mathrm{~mm}$, que acompanha a prensa hidráulica UTM-25 da IPC Global. Na Figura 2 pode-se observar o esquema de posicionamento dos LVDTs e a unidade controladora do ensaio.

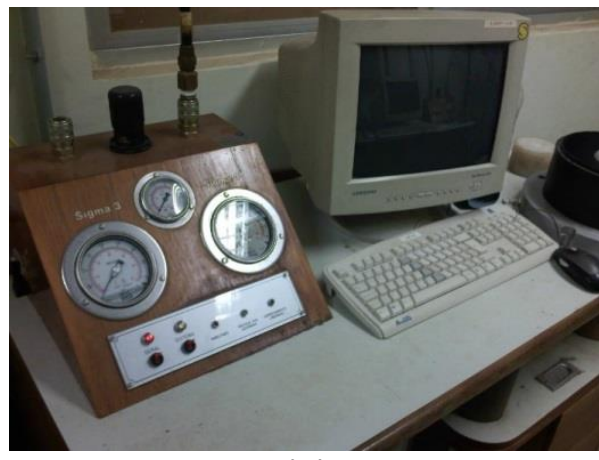

(b)

Figura 2 - (a) Esquema de posicionamento dos LVDTs no ensaio de MR e (b) Unidade controladora do ensaio

O controle da temperatura de ensaio e o período de estabilização foram realizados na capela do próprio equipamento de ensaio. Para isso, controlou-se tanto a temperatura da câmara quanto do interior do CP. Isso foi possível com a utilização de dois termômetros, um medindo a temperatura da câmara, e outro instalado no interior de um CP, posicionado dentro da câmara, mas que não foi ensaiado.

\subsection{Tratamento dos resultados provenientes dos ensaios de MR}

Os cálculos dos valores de MR para as amostras ensaiadas seguindo as metodologias das normas DNIT ME 133 (1994) e ABNT NBR 16018 (2011) foram realizados através de uma planilha no Excel desenvolvida para a obtenção do deslocamento instantâneo dos ciclos utilizados para o cálculo do MR de acordo com a me- 
todologia utilizada para a realização do ensaio. Para a metodologia usada pelo equipamento da SIGEO (SICAEP, 2004) os valores de MR foram obtidos diretamente do software do equipamento.

A obtenção dos deslocamentos instantâneos e totais para o cálculo do MR é realizada através da subdivisão do pulso de carregamento em: (i) pico de deslocamento, (ii) porção reta do caminho de descarregamento, (iii) porção de recuperação e (iv) uma porção curva que une o caminho de descarregamento à porção de recuperação. A divisão dessas porções é feita através de regressões lineares e hiperbólicas, tangentes à curva do pulso de deslocamento. Uma explicação mais aprofundada sobre a me- todologia da subdivisão do pulso de carregamento e da definição dos deslocamentos instantâneos e totais pode ser encontrada em Bernucci et al. (2007). A Figura 3 traz a representação gráfica da subdivisão do pulso de deslocamento durante o ensaio de MR, enquanto a Figura 4 ilustra os deslocamentos instantâneo e total.

A norma DNER ME 133 (1994) não especifica a metodologia para obtenção dos deslocamentos instantâneo e total. Utilizou-se, então, a metodologia apresentada na norma ABNT NBR 16018 (2011), similar à apresentada na norma americana AASHTO TP31 (1996), evidenciado o alinhamento da nova norma brasileira em relação às normas internacionais.

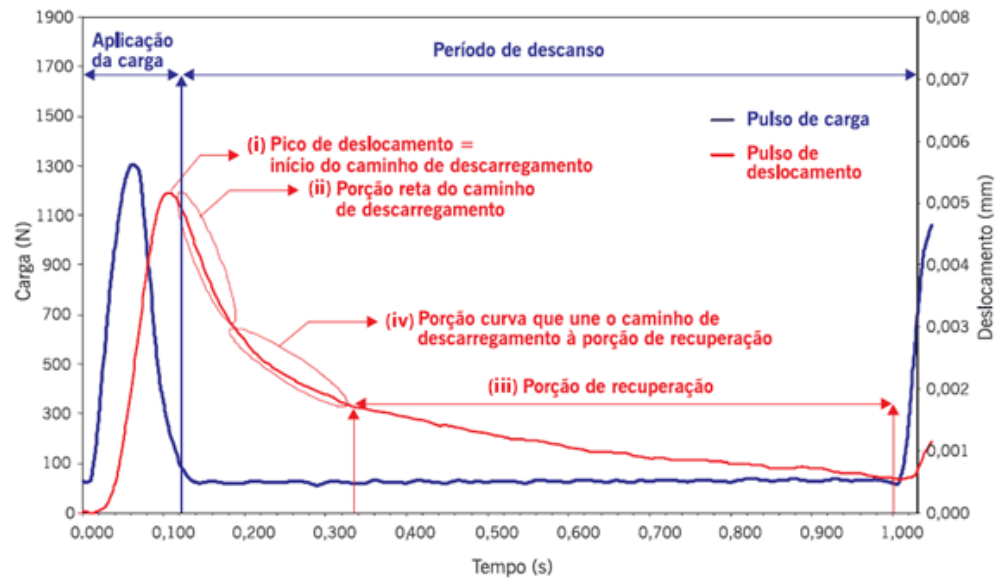

Figura 3 - Divisão do pulso de deslocamento - Adaptado de Bernucci et al. (2007)

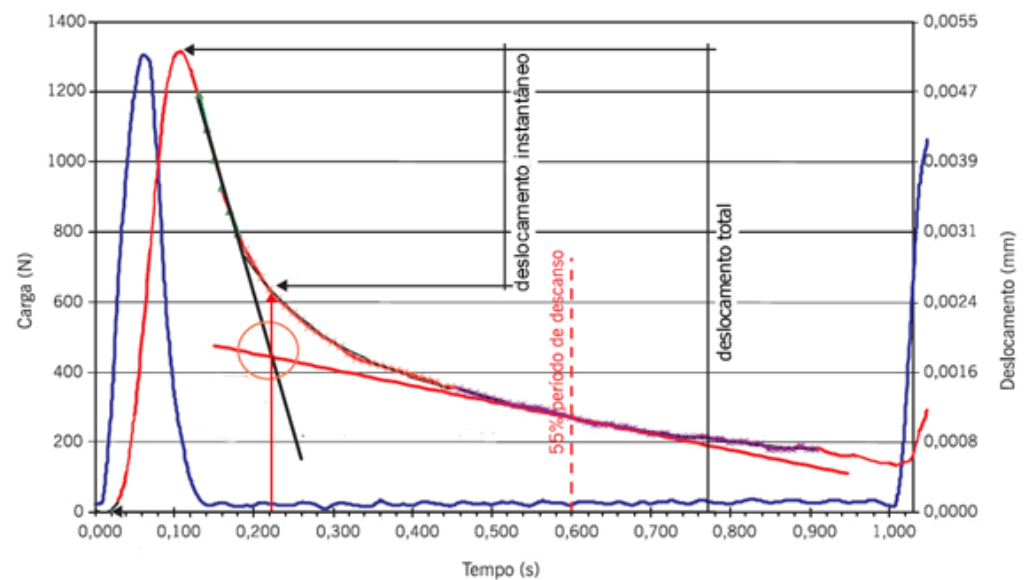

Figura 4 - Definições de deslocamento instantâneo e total para o cálculo do MR para misturas asfálticas - Adaptado de Bernucci et al. (2007)

\section{RESULTADOS E ANÁLISES}

\subsection{Resultados dos ensaios de MR}

Foram realizados ensaios nas Misturas 1, 2 e 3 utilizando-se as metodologias previamente descritas, na prensa pneumática (SIGEO). Seguem, abaixo nas Tabelas 2, 3 e 4, os resultados de MR obtidos em MPa, assim como os valores de desvio padrão e Coeficiente de Variação (CV) para as amostras avaliadas. 
Tabela 2 - Resultados de MR - Prensa pneumática: metodologia SIGEO (SICAEP, 2004)

\begin{tabular}{|c|c|c|c|c|c|c|c|c|}
\hline \multicolumn{3}{|c|}{ Mistura 1} & \multicolumn{3}{|c|}{ Mistura 2} & \multicolumn{3}{|c|}{ Mistura 3} \\
\hline $\mathrm{CP}$ & Vv (\%) & MR (MPa) & CP & Vv (\%) & $\mathrm{MR}(\mathrm{MPa})$ & $\mathrm{CP}$ & Vv (\%) & $\mathrm{MR}(\mathrm{MPa})$ \\
\hline 1 & 4,0 & 4370 & 1 & 4,3 & 3724 & 1 & 4,4 & 4563 \\
\hline 2 & 3,6 & 4247 & 2 & 4,3 & 3753 & 2 & 4,4 & 5371 \\
\hline 3 & 4,0 & 3601 & 3 & 3,9 & 3736 & 3 & 4,3 & 5702 \\
\hline 4 & 4,1 & 4232 & 4 & 4,0 & 3896 & 4 & 4,3 & 5270 \\
\hline 5 & 4,0 & 4076 & 5 & 3,8 & 3911 & 5 & 4,4 & 5367 \\
\hline & médio & 4105 & & médio & 3804 & & médio & 5255 \\
\hline & Padrão & 301 & & Padrão & 92 & & padrão & 420 \\
\hline & $(\%)$ & 7 & & (\%) & 2 & & $(\%)$ & 8 \\
\hline
\end{tabular}

Tabela 3 - Resultados de MR - Prensa pneumática: metodologia ABNT NBR 16018 (2011)

\begin{tabular}{|c|c|c|c|c|c|c|c|c|}
\hline \multicolumn{3}{|c|}{ Mistura 1} & \multicolumn{3}{|c|}{ Mistura 2} & \multicolumn{3}{|c|}{ Mistura 3} \\
\hline $\mathrm{CP}$ & $\mathrm{Vv}(\%)$ & $\mathrm{MR}(\mathrm{MPa})$ & $\mathrm{CP}$ & Vv (\%) & $\mathrm{MR}(\mathrm{MPa})$ & $\mathrm{CP}$ & Vv (\%) & $\mathrm{MR}(\mathrm{MPa})$ \\
\hline 1 & 4,0 & 4309 & 1 & 4,3 & 3786 & 1 & 4,4 & 5105 \\
\hline 2 & 3,6 & 3970 & 2 & 4,3 & 3878 & 2 & 4,4 & 5273 \\
\hline 3 & 4,0 & 4243 & 3 & 3,9 & 4184 & 3 & 4,3 & 4916 \\
\hline 4 & 4,1 & 3867 & 4 & 4,0 & 4170 & 4 & 4,3 & 5434 \\
\hline 5 & 4,0 & 4351 & 5 & 3,8 & 4346 & 5 & 4,4 & 5127 \\
\hline & Rédio & 4148 & \multicolumn{2}{|c|}{ MR médio } & 4073 & \multicolumn{2}{|c|}{$\mathrm{MR}_{\text {médio }}$} & 5171 \\
\hline & Padrão & 216 & \multicolumn{2}{|c|}{ Desv. Padrão } & 233 & \multicolumn{2}{|c|}{ Desv. padrão } & 194 \\
\hline & (\%) & 5 & \multicolumn{2}{|c|}{ CV (\%) } & 6 & \multicolumn{2}{|c|}{ CV (\%) } & 4 \\
\hline
\end{tabular}

Tabela 4 - Resultados de MR - Prensa pneumática: metodologia DNER ME 133 (1994)

\begin{tabular}{|c|c|c|c|c|c|c|c|c|}
\hline \multicolumn{3}{|c|}{ Mistura 1} & \multicolumn{3}{|c|}{ Mistura 2} & \multicolumn{3}{|c|}{ Mistura 3} \\
\hline $\mathrm{CP}$ & Vv (\%) & MR (MPa) & CP & Vv (\%) & MR (MPa) & CP & Vv (\%) & MR (MPa) \\
\hline 1 & 4,0 & 4108 & 1 & 4,3 & 3778 & 1 & 4,4 & 4864 \\
\hline 2 & 3,6 & 3846 & 2 & 4,3 & 3595 & 2 & 4,4 & 5156 \\
\hline 3 & 4,0 & 4227 & 3 & 3,9 & 3762 & 3 & 4,3 & 4651 \\
\hline 4 & 4,1 & 3434 & 4 & 4,0 & 3718 & 4 & 4,3 & 4986 \\
\hline 5 & 4,0 & 3892 & 5 & 3,8 & 3603 & 5 & 4,4 & 4902 \\
\hline & Zmédio & 3901 & & Zmédio & 3691 & & Imédio & 4912 \\
\hline & padrão & 304 & & Padrão & 87 & & padrão & 184 \\
\hline & (\%) & 8 & & (\%) & 3 & & $(\%)$ & 4 \\
\hline
\end{tabular}

Na Tabela 5 encontra-se o resumo dos valores de MR encontrados para as três misturas avaliadas utilizando-se as metodologias especificadas. Nesta Tabela consta a média dos valores de MR obtidos através da utilização das metodo- logias em estudo, o valor médio de MR considerando as 15 amostras ensaiadas, como também, o desvio padrão entre os resultados encontrados e os respectivos CVs.

Tabela 5 - Resumo dos resultados de MR (MPa) obtidos em prensa pneumática para metodologias distintas

\begin{tabular}{ccccccc}
\hline Mistura/Norma & $\begin{array}{c}\text { SIGEO } \\
\text { (SICAEP,2004) }\end{array}$ & $\begin{array}{c}\text { ABNT NBR 16018 } \\
\mathbf{( 2 0 1 1 )}\end{array}$ & $\begin{array}{c}\text { DNER ME 133 } \\
\text { (1994) }\end{array}$ & Média & $\begin{array}{c}\text { Desv. Pa- } \\
\text { drão }\end{array}$ & $\begin{array}{c}\text { CV (\%) } \\
\text { Mistura 1 }\end{array}$ \\
Mistura 2 & 4105 & 4148 & 3901 & 4052 & 174 & 4 \\
Mistura 3 & 3804 & 4073 & 3691 & 3856 & 270 & 7 \\
\hline
\end{tabular}

Pode-se observar na Tabela 5, que os resultados de MR obtidos através do uso das três metodologias estudadas foram semelhantes, apresentando CV mínimo de $4 \%$ e máximo de $7 \%$. Considerando fatores como heterogeneidade dos materiais e fatores humanos na montagem e realização do ensaio, acredita-se que as três metodologias levaram praticamente ao mesmo resultado para todas as misturas avaliadas.
Os resultados obtidos através do uso da norma DNER ME 133 (1994) apresentaram os menores valores de MR para as três misturas ensaiadas. Esse comportamento já era esperado, visto que a temperatura de ensaio recomendada é de $30^{\circ} \mathrm{C}$ (superior à temperatura de ensaio de $25^{\circ} \mathrm{C}$ sugerida pelas outras metodologias), acarretando na diminuição da viscosidade do ligante na amostra, e, consequentemente, no aumento das deformações computadas durante o ensaio. 
Dos resultados obtidos pode-se observar que, quanto à repetibilidade, os resultados de MR obtidos através do uso da metodologia da SIGEO apresentaram o maior $\mathrm{CV}_{\text {médio }}$ (Tabela 6) entre as metodologias estudadas, como também, juntamente à norma DNER ME 133 (1994), as maiores amplitudes (diferença entre o maior e o menor valor de $\mathrm{CV}$ ). Os resultados obtidos através do uso da norma ABNT NBR 16018 (2011) mostraram maior constância, o que é evidenciado pela menor amplitude dos valores encontrados através do uso dessa metodologia, para todas as misturas avaliadas nesse estudo.

Tabela 6 - Coeficientes de Variação (CV) dos resultados de MR encontrados para cada mistura para as três metodologias estudadas

\begin{tabular}{cccccc}
\hline Norma/Mistura & $\begin{array}{c}\text { CV(\%) } \\
\text { Mistura 1 }\end{array}$ & $\begin{array}{c}\text { CV(\%) } \\
\text { Mistura 2 }\end{array}$ & $\begin{array}{c}\text { CV(\%) } \\
\text { Mistura 3 }\end{array}$ & Média (\%) & Amplitude (\%) \\
\hline SIGEO (SICAEP, 2004) & 7 & 2 & 8 & 6 & 6 \\
ABNT NBR 16018 (2011) & 5 & 6 & 4 & 5 & 2 \\
DNER ME 133 (1994) & 8 & 2 & 4 & 5 & 6 \\
\hline
\end{tabular}

\subsection{Impacto do uso de diferentes metodolo- gias para obtenção do MR na análise de um pavimento padrão}

Para a avaliação da influência que a variação do valor de MR de misturas asfálticas exerce no comportamento do pavimento como um todo, não apenas na mistura asfáltica em si, utilizouse o software CAP3D (Holanda et al., 2006) para a análise das tensões e das deformações das camadas do pavimento. Este software, desenvolvido no LMP/UFC, é um programa para análise de pavimentos asfálticos através do Método dos Elementos Finitos (MEF), atualmente, sendo capaz de tratar modelos planos, axissimétricos e tridimensionais, utilizando elementos de diferentes formas (triângulos, quadriláteros, tetraedros e hexaedros) e ordens de interpolação (linear e quadrática) (Bastos et al., 2012).

Para a análise, adotou-se um pavimento padrão constituído de revestimento e base, assentados sob o subleito. A geometria adotada para a análise, as características dos materiais granulares e o carregamento axissimétrico $(550 \mathrm{kPa})$ aplicado foi semelhante aquele utilizado no estudo de Silva e Holanda (2011). Para a modelagem do problema, utilizou-se uma malha mista, onde as camadas de revestimento e de base foram modeladas com uma malha de elementos finitos, enquanto o subleito foi modelado como uma camada de elementos finitos e infinitos, para melhor representação da realidade. A Figura 5 traz uma representação do pavimento padrão utilizado para a análise e suas características.

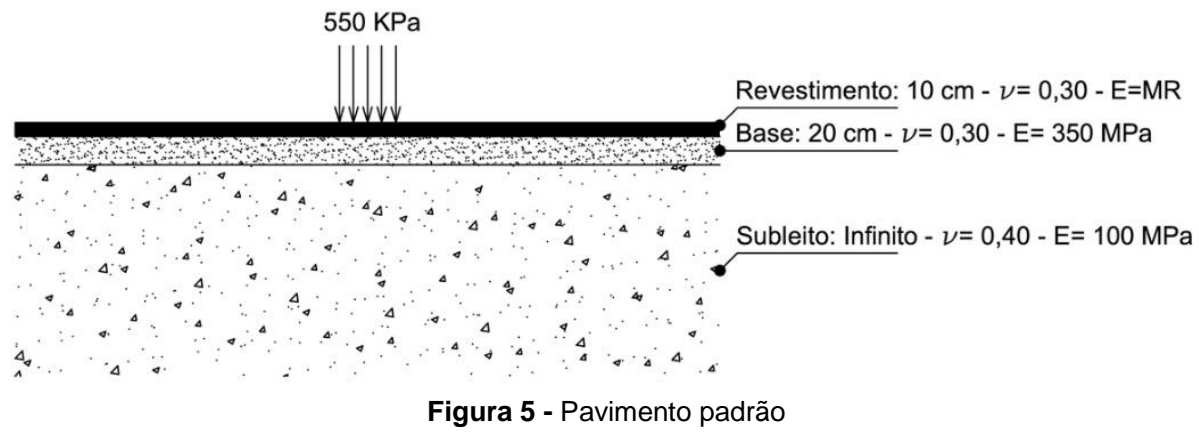

Foram realizadas nove análises para a estrutura do pavimento padrão, visto que estão sendo estudadas três metodologias de ensaio e três misturas asfálticas. Para a análise, mantiveram-se constante as espessuras das camadas e as características das camadas granulares (coeficiente de Poisson e Módulo de Elasticidade (E)) e variou-se as características da camada de revestimento. Para os dados de entrada referentes à camada de revestimento considerou-se E=MR (ou seja, foi realizada uma análise elástica linear) e adotou-se o coeficiente de Poisson assumido por cada metodologia em estudo. Como todas as metodologias recomendam que se utilize $v=0,30$ (quando não há possibilidade de se obtê-lo), este manteve-se constante para todas as análises realizadas. 
Para a análise do pavimento padrão realizada neste trabalho foram observados valores de tensões e deslocamentos em pontos críticos da estrutura, quais sejam: (i) deslocamento vertical na superfície do revestimento, (ii) tensão de tração na fibra inferior do revestimento e (iii) tensão de compressão no topo do subleito. Estes critérios buscam assegurar bom desempenho ao pavimento em relação à vida de fadiga e à resistência à deformação permanente. Os resultados obtidos estão representados na Figura 6.

A partir da análise dos três critérios observados, pode-se inferir que a resposta do pavimento foi muito similar para os critérios de deslocamento vertical na superfície do revestimento e de tensão de compressão no topo do subleito (Figuras 6a e 7). Para o critério de deslocamento vertical na superfície do revestimento, foram obtidos os seguintes CVs: (i) Mistura 1: 0,7\%, (ii) Mistura 2: 0,9\% e (iii) Mis- tura 3: $0,7 \%$, enquanto no critério de tensão de compressão no topo do subleito os CVs foram: : (i) Mistura 1: 0,9\%, (ii) Mistura 2: 1,5\% e (iii) Mistura 3: 1,0\%. Esses resultados levam a crer que, para esses quesitos, que buscam representar o desempenho do pavimento quanto à resistência a deformação permanente, para a estrutura avaliada, não houve influência considerável da metodologia aplicada para obtenção do MR para as misturas asfálticas.

O critério analisado que apresentou maior sensibilidade à variação dos valores de MR adotados para a camada de revestimento foi o de tensão de tração na fibra inferior do revestimento (Figura 6b). Os CVs obtidos foram: (i) Mistura 1:2,5\%, (ii) Mistura 2: 3,9\% e (iii) Mistura 3: 2,4\%. Porém, pode-se considerar também que não houve variação significativa para este quesito.

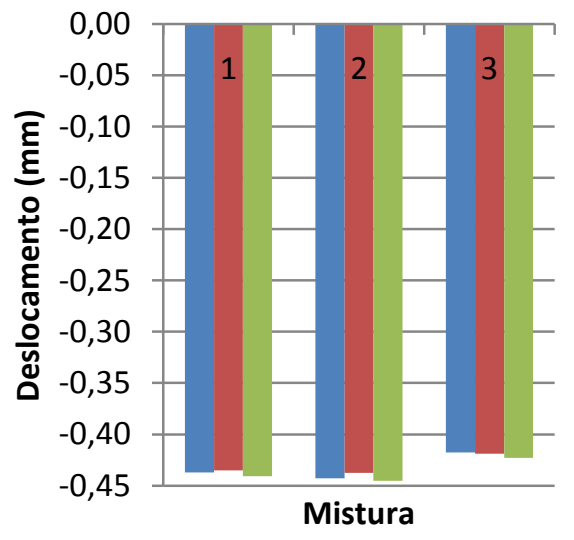

(a)

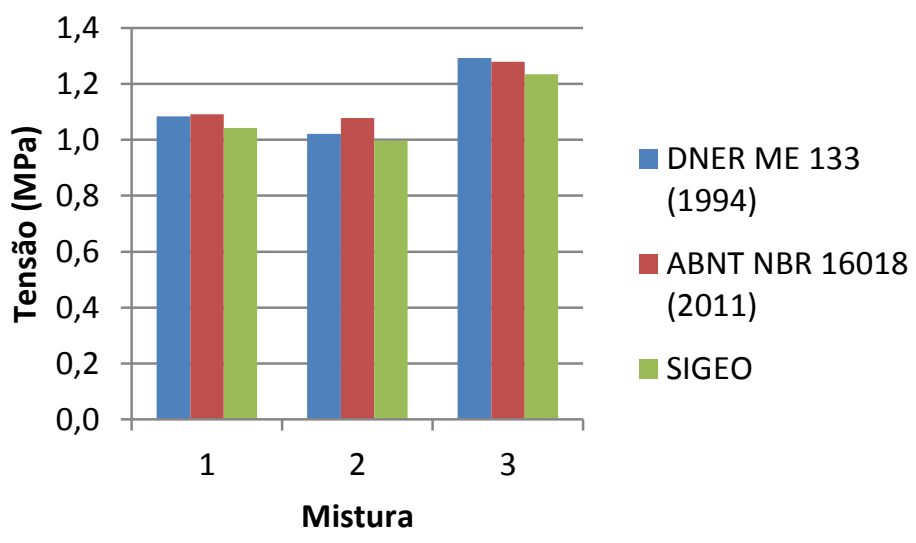

(b)

Figura 6 - Análises: (a) deslocamento vertical $(\mathrm{mm})$ na superfície do revestimento, (b) tensão de tração (MPa) na face inferior do revestimento

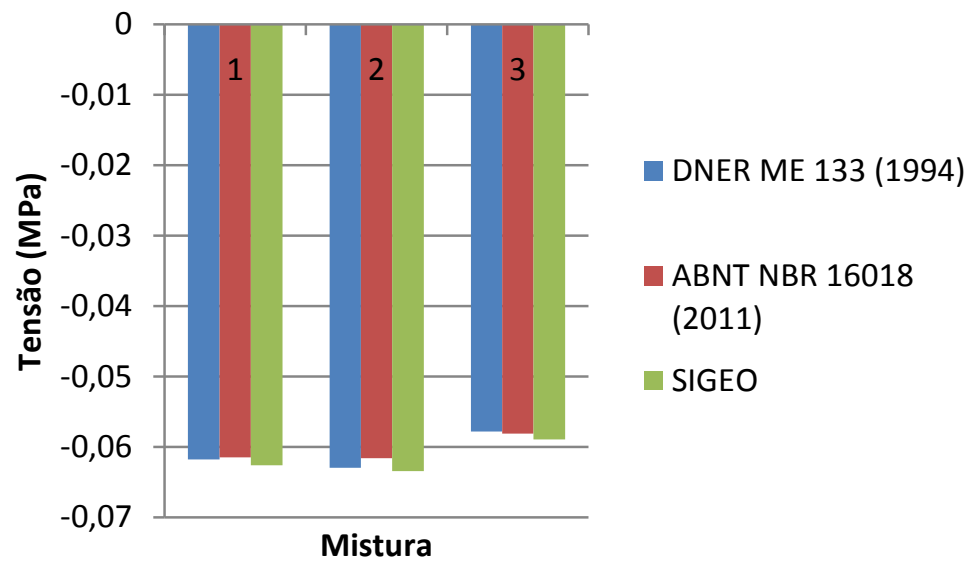

Figura 7 - Análise: Tensão de compressão no topo do subleito (MPa)

\section{CONCLUSÕES}

Neste trabalho realizou-se uma avaliação da influência de diferentes metodologias para a obtenção do MR para misturas asfálticas. Foi realizado um levantamento das principais norma- 
tivas nacionais e internacionais e observou-se que estas divergem em diversas recomendações para a realização do ensaio, como por exemplo: temperatura de ensaio, número de ciclos de précondicionamento e número de ciclos de condicionamento.

Dentre as normas nacionais, a ABNT NBR 16018 (2011) possui o texto mais específico, sendo a única que descreve os procedimentos para a obtenção dos deslocamentos utilizados para o cálculo do MR, como também traz recomendações para a obtenção do coeficiente de Poisson, tratamento dos dados quando se faz diferentes montagens de LVDTs, incluindo ainda o cálculo do MR total. Esta norma foi elaborada baseada nas principais metodologias internacionais, como a ASTM D 7369 (2009) e a EN 12697-26 (2003).

A outra norma brasileira em vigor, DNIT ME 135 (2010), não traz recomendações para diversos parâmetros de ensaio como: frequência de carregamento, número de ciclos de pré-condicionamento e número de ciclos de condicionamento e período para estabilização da temperatura de ensaio. A utilização dessa norma para a realização do ensaio de MR para misturas asfálticas deixa a cargo do operador decisões sobre parâmetros que podem vir a influenciar os resultados. Desta forma, não se recomenda o uso desta norma para a realização do ensaio.

A partir dos resultados dos ensaios de MR para misturas asfálticas realizados em prensa pneumática, através do uso das metodologias SIGEO (SICAEP, 2004), DNIT ME 133 (1994) e ABNT NBR 16018 (2011) concluiu-se que para as misturas do tipo CA avaliadas nesse estudo não houve influência da metodologia de ensaio nos resultados obtidos. Observou-se, porém, que, o parâmetro que levou a uma maior variação nos resultados foi a temperatura.

Dentre as metodologias ensaiadas, a que apresentou melhor consistência foi a ABNT NBR 16018 (2011). Os resultados provenientes da aplicação desta metodologia apresentaram menor sensibilidade no quesito repetibilidade quanto à mistura ensaiada, como também menor dispersão dos resultados obtidos para cada mistura. Devido a estes fatores, como também, a maior especificidade do texto, recomenda-se a utilização desta norma para a realização de ensaios de MR para misturas asfálticas no Brasil.

Da análise de um pavimento padrão, através do software CAP $3 D$, onde os valores de MR foram utilizados como dados de entrada para a rigidez da camada de revestimento, concluiu-se que não houve influência significativa da metodologia utilizada para a obtenção do MR para misturas asfálticas no resultado da análise de tensões e de deformações deste pavimento.

Todos os ensaios realizados para a elaboração deste trabalho foram executados em equipamento pneumático. Recomenda-se a continuidade deste estudo considerando diferentes prensas, utilização de carregamento hidráulico e a utilização de coeficiente de Poisson determinado.

\section{REFERÊNCIAS}

AASHTO (1996) TP 31-96 - Standard Method of Test for Determining Resilient Modulus of Bituminous Mixtures by Indirect Tension. In: AASHTO Provisional Standard TP-31-96 Edition 1B. Washington, D.C.

ABNT (2011) NBR 16018 - Misturas asfálticas - Determinação da rigidez por compressão diametral sob carga repetida. Rio de Janeiro.

ALENCAR, A. E. V. (2009) Avaliação do Uso de Polímero EVA, Resíduo da Indústria de Calçados (EVAR) e Aditivos no Desempenho Reológico de Ligante Asfáltico de Petróleo. 2009. Tese (Doutorado em Química) - Programa de Pós-Graduação em Química da Universidade Federal do Ceará, Fortaleza.

ARAÚJO, P. C. J.; FERREIRA, W. L. G; CASTELO BRANCO, V. T. F e J. B. SOARES (2009) Considerações sobre o Ensaio de Compressão Diametral no estudo de Materiais de Pavimentação. In: XXIII Congresso de Pesquisa e Ensaio em Transportes, ANPET, Vitória.

ASTM (1995) D 7369 - Standard Test Method for Determining the Resilient Modulus of Bituminous Mixtures by Indirect Tension Test. Philadelfia.

AUSTRALIAN STANDARD (1995) AS 2891.13.1 - Methods of sampling and testing asphalt. Method 13.1: Determination of the resilient modulus of asphalt - Indirect tensile method. Sidney.

BASTOS, J. B. S.; HOLANDA, A. S. e S. H. A. BARROSO (2012) Influência da Variação da Umidade na Análise dos Pavimentos da Região Metropolitana de Fortaleza. In: XVI CONGRESSO

BERNUCCI, L. B.; MOTTA, L. M. G.; CERATTI, J. A. P. e J. B. SOARES (2007) Pavimentação Asfáltica - Formação Básica para Engenheiros. 1. ed. Rio de Janeiro: PETROBRAS: ABEDA.

BESSA, I. S. (2012) Avaliação do processamento digital de imagens como ferramenta para caracterização de agregados $e$ misturas asfálticas. Dissertação (Mestrado em Engenharia Civil) - Programa de Mestrado em Engenharia de Transportes, Universidade Federal do Ceará, Fortaleza.

BRASILEIRO DE MECÂNICA DOS SOLOS E ENGENHARIA GEOTÉCNICA, 2012, Porto de Galinhas. Anais... XVI Congresso Brasileiro de Mecânica dos Solos e Engenharia Geotécnica, Porto de Galinhas. 
BRITO, L. A. T. (2006) Avaliação e análise paramétrica do ensaio de compressão diametral sob cargas repetidas em misturas asfálticas. Dissertação (Mestrado em Engenharia Civil) - Programa de Pós-Graduação em Engenharia Civil Universidade Federal do Rio Grande do Sul, Porto Alegre.

BSI (1993) DD213: Determination of the Indirect Tensile Stiffness Modulus of Bituminous Mixtures. Londres.

COUTINHO, R. P (2012) Utilização da parte fina de misturas asfálticas para avaliação do dano por fadiga. Dissertação (Mestrado em Engenharia Civil) - Programa de Mestrado em Engenharia de Transportes, Universidade Federal do Ceará, Fortaleza.

DNER (1994) ME 133 - Misturas Betuminosas - Determinação do Módulo de Resiliência - Método de Ensaio. Rio de Janeiro.

DNIT (2006) ES 031- Pavimentos flexíveis - Concreto asfáltico - Especificação de serviço. Rio de Janeiro.

DNIT (2010) ME 135 - Pavimentação asfáltica - Misturas asfálticas - Determinação do módulo de resiliência - Método de ensaio. Rio de Janeiro.

DORMON, G. M. e C. T. METCALF (1965) Design Curves for Flexible Pavements Based on Layered System Theory. Highway Research Record 71, Highway Research Board. Washington, D.C.

EUROPEAN STANDARD (2003) EN 12967-26 - Bituminous mixtures - Test methods for hot mix asphalt - Part 26: Stiffness. European Committee for Standardization.

FRANCO, F. A. C. P (2007) Método de dimensionamento mecanístico-empírico de pavimentos asfálticos - SisPav. Tese (Doutorado em Engenharia Civil) - Universidade Federal do Rio de Janeiro, Rio de Janeiro.
HOLANDA, A. S.; PARENTE JR., E.; ARAÚJO, T. D. P.; MELO, L. T. B.; EVANGELISTA JR., F. e J. B. SOARES (2006) Finite Element Modeling of Flexible Pavements. XXVII Iberian Latin American Congress on Computational Methods in Engineering, CILAMCE, Belém.

KERKHOVEN, R. E. e G. M. DORMON (1953) Some Considerations on the California Bearing Ratio Method for the Design of Flexible Pavements. Shell Bitumen Monograph $\mathrm{N}^{\mathrm{o}} 1$, Amsterdam.

MOTTA, L. M. G (1991) Método de dimensionamento de pavimentos flexíveis; Critério de confiabilidade e ensaios de cargas repetidas. 1991. Tese (Doutorado em Engenharia Civil) - Programa de Pró-Graduação da Universidade Federal do Rio de Janeiro, Rio de Janeiro.

SAAL, R. N. e P. S. PELL (1960) Fatigue of bituminous road mixes. Kolloid-Zeitschrift. 171 p.

SICAEP (2004) - Manual do Usuário do Sistema de Controle e Aquisição de dados para Ensaios de Pavimentos. SIGEO. Rio de Janeiro.

SILVA, S. A. T. e A. S. HOLANDA (2011) Uso de elementos infinitos na análise de pavimentos flexíveis. In: AMOSTRA CIENTÍFICA DE PESQUISAS DA ENGENHARIA CIVIL, 5. Fortaleza.

SOARES, J. B.; MORENO, A. M. e MOTTA, L. M. G. (2009) Aspectos gerais de Métodos de Dimensionamento de Pavimentos Asfálticos de vários países e a relação com um novo método brasileiro. Revista Pavimentação, v. XVII, p. 20-35. 